\title{
A New Diabetic Mouse Model Derived from the ddY Strain
}

\author{
Ichiro Noge, Yoshiyuki Kagawa, and Toshio MaedA* \\ Department of Clinical Pharmaceutics \& Pharmacy Practice, Graduate School of Pharmaceutical Sciences, University of \\ Shizuoka; 52-1 Yada, Suruga-ku, Shizuoka 422-8526, Japan. \\ Received December 25, 2009; accepted March 24, 2010; published online March 31, 2010
}

Selective breeding was used to isolate hyperglycemic (ddY-H) or normoglycemic (ddY-L) mice that had been induced by fasting and refeeding. Serum glucose levels $12 \mathrm{~h}$ after $48 \mathrm{~h}$-fasting were high in male ddY-H mice, but relatively low in male ddY-L mice compared with control ddY mice. Glucose tolerance was impaired in ddY-H mice maintained with standard chow pellets ad libitum at 12 weeks of age, and serum glucose and insulin levels were significantly increased after overnight fasting at 15 weeks of age. ddY-L mice indices did not differ from ddY mice indices, suggesting that insulin resistance is spontaneously induced in ddY-H mice. Increases in urinary excretion and urinary sugar accompanied by increased body mass were observed in all ddY-H mice, but not in ddY or ddY-L mice, at 27 weeks of age, indicating the induction of diabetic symptoms. Cross-mating between ddY-H and ddY-L mice was used to certify the genetic involvement in impaired glucose tolerance. This was not induced in mice born from male ddY-H and female ddY-L mice, or from female ddY-H and male ddY-L mice. In conclusion, ddY-H mice are a useful diabetic mouse model that show spontaneously induced insulin resistance followed by diabetic symptoms that are maintained by standard chow pellets. Their characteristics are recessively inherited, and ddY-L mice are an appropriate choice as control mice.

Key words diabetic mouse model; hyperinsulinemia; insulin resistance

Hyperglycemia is a persistent symptom of diabetes mellitus. The complex pathogenesis of type 2 diabetes involves the progressive development of insulin resistance and defective insulin secretion, which leads to overt hyperglycemia. ${ }^{1)}$ These pathological states are mostly attributable to life styles and genetic background, ${ }^{2)}$ and various animal models of diabetes have been developed and investigated to clarify the mechanisms by which hyperglycemia is induced..$^{3-8)}$

We previously showed that sustained hyperglycemia could be induced in ddY mice by refeeding with standard chow pellets after fasting for $48 \mathrm{~h} .{ }^{9)}$ We proposed that, in this case, hyperglycemia was induced by defects in insulin secretion and action, and maintained by the resulting insulin resistance. However glucose levels and the duration of hyperglycemia were variable in individual mice. Since then, we have attempted to select those mice with induced serious hyperglycemia by selective breeding based on serum glucose levels after refeeding. Two strains, namely, spontaneous insulin resistant mice (ddY-H) and none insulin resistant mice (ddY-L), have been isolated from ddY mice by inbreeding. In this study, we investigated the characteristics of these mice.

\section{MATERIALS AND METHODS}

Animal Care Four-week-old male ddY mice were purchased from SLC Inc. (Hamamatsu, Japan), and ddY-H and ddY-L mice from our own colony were used. Mice were maintained on $12 \mathrm{~h} \mathrm{light/dark} \mathrm{cycles} \mathrm{with} \mathrm{free} \mathrm{access} \mathrm{to} \mathrm{stan-}$ dard chow pellets (MF diet, Oriental Yeast Co., Ltd., Tokyo, Japan) and water ad libitum until the experiments were carried out. Animal care and experiments were performed in accordance with the guidelines for the care and use of laboratory animals of the University of Shizuoka, Japan.

Fasting and Refeeding Fasting commenced by removing the chow pellets from mouse home cages at 21:00. After $48 \mathrm{~h}$, mice were refed standard chow pellets for $12 \mathrm{~h}$ and blood was obtained from the caudal vein for the determina- tion of serum glucose level. In some experiments, mice were fasted for $12 \mathrm{~h}$ and refed for $1 \mathrm{~h}$ as described above.

Oral Glucose Tolerance Oral glucose tolerance test was carried out as described previously. ${ }^{9)}$ Briefly, mice were orally administered with glucose $(3 \mathrm{~g} / \mathrm{kg})$ and blood was withdrawn from caudal veins $0,30,60$ and 120 min later to measure serum glucose level.

Measurement of Serum Glucose and Insulin Levels Serum glucose and insulin levels were determined using Glucose CII Test Wako (Wako Pure Chemical Industries, Ltd., Osaka, Japan) and the Insulin ELISA kit (Morinaga Institute of Biological Sciences, Inc., Kanagawa, Japan), respectively, according to manufacturer's instructions.

Insulin Sensitivity and Insulin Resistance Insulin sensitivity and insulin resistance were calculated from glucose and insulin levels in the serum of mice fasted for $12 \mathrm{~h}$ (21:00 - 9:00) as follows: glucose ( $\mathrm{mg} / \mathrm{dl}) /$ insulin $(\mathrm{ng} / \mathrm{dl})$ and glucose $(\mathrm{mg} / \mathrm{dl}) \times$ insulin $(\mathrm{ng} / \mathrm{dl}) / 100$, respectively. ${ }^{10,11)}$

Cross-Mating between ddY-H Mice and ddY-L Mice ddY-H mice and ddY-L mice at 10 weeks of age were crossmated. Newborn mice from male ddY-H and female ddY-L and from female ddY-H and male ddY-L mice were named as ddY-HL and ddY-LH, respectively. Newborn mice were weaned at 4 weeks of age and were maintained with standard chow pellets ad libitum for the glucose tolerance test at 12 weeks of age.

Data and Statistical Analysis All data are expressed as mean \pm S.D. of eight to 12 mice. A variance was analyzed by Kruskal-Wallis non-parametric ANOVA test and each group was compared using the Tukey's post hoc test. Statistical significance was set at $p<0.05$.

\section{RESULTS}

Serum Glucose after Refeeding in ddY-H and ddY-L Mice As described previously, sustained hyperglycemia was observed in ddY mice that were refed after fasting for 


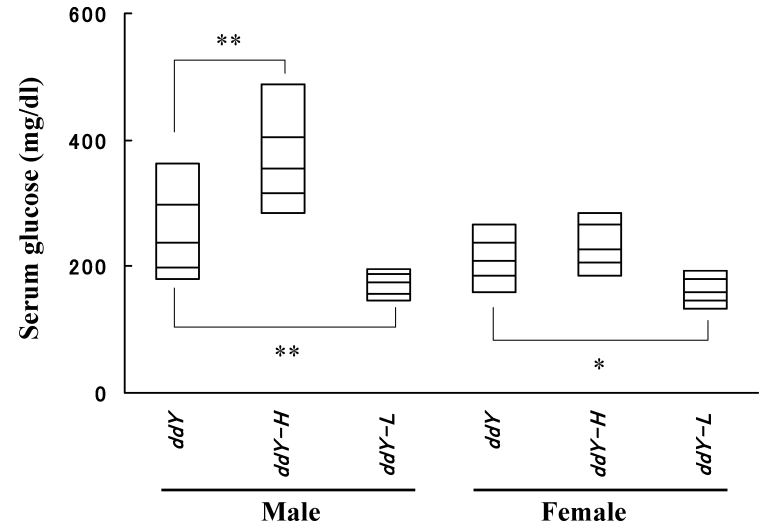

Fig. 1. Serum Glucose Level after Refeeding

Mice fasted for $48 \mathrm{~h}$ were refed standard chow pellets for $12 \mathrm{~h}$ and serum glucose level was measured. Boxes represent 10, 25, 50, 75, and 90 percentiles of 20 mice. $* * p<0.01$ vs. ddY, $* p<0.05$ vs. ddY.

$48 \mathrm{~h} .{ }^{9)}$ However, the glucose levels varied between individual mice (Fig. 1). Mice of both genders showing the highest and lowest glucose levels after refeeding were selected. The male and female mice with the highest glucose levels were mated, and selective breeding was repeated for 20 generations by measuring glucose levels after refeeding as an index. Offspring were named as ddY-H. Similar selective breeding of mice with the lowest glucose levels after refeeding produced offspring named ddY-L.

Figure 1 shows serum glucose levels in ddY, ddY-H, and ddY-L mice refed standard chow pellets for $12 \mathrm{~h}$ ad libitum after 48-h fasting. Serum glucose level was significantly higher in male ddY-H mice (median, $356 \mathrm{mg} / \mathrm{dl}$ ) and significantly lower in male ddY-L mice (median, $175 \mathrm{mg} / \mathrm{dl}$ ) than in male ddY mice (median, $238 \mathrm{mg} / \mathrm{dl}$ ). The significant difference between male ddY-H and male ddY-L mice suggested that the two strains that responded differently to refeeding had been successfully isolated. On the other hand, differences in serum glucose level between female ddY (median, $208 \mathrm{mg} / \mathrm{dl}$ ), ddY-H (median, $235 \mathrm{mg} / \mathrm{dl}$ ) and ddY-L (median, $160 \mathrm{mg} / \mathrm{dl}$ ) mice were not remarkable. The dietary intakes during refeeding were $5.8 \mathrm{~g} /$ mouse (ddY), $5.7 \mathrm{~g} / \mathrm{mouse}$ (ddY$\mathrm{H})$, and $6.0 \mathrm{~g} / \mathrm{mouse}$ (ddY-L) for male mice, and $5.0 \mathrm{~g} / \mathrm{mouse}$ (ddY), $4.9 \mathrm{~g} / \mathrm{mouse}$ (ddY-H), and $5.1 \mathrm{~g} /$ mouse (ddY-L) for female mice, indicating that intake did not contribute to differences in serum glucose level.

Serum Glucose after Maintaining with Standard Chow Pellets Male mice were maintained with standard chow pellets and, at 5, 9, 15, and 20 weeks of age, serum glucose levels were measured after overnight fasting or refeeding with standard chow pellets for the following $1 \mathrm{~h}$. Until 9 weeks of age, there were no differences in serum glucose level after fasting and refeeding between ddY and ddY-H mice. ddY-H mice serum glucose level was significantly higher than those of ddY and ddY-L mice at 15 and 20 weeks of age (Fig. 2). Compared with ddY mice, ddY-L mice showed a low tendency of serum glucose after fasting and refeeding (Fig. 2). As an index of dietary intake during refeeding, the gastric contents were measured in ddY, ddY-H, and ddY-L mice of all ages, but no differences were found (data not shown). This suggests that the elevated blood glucose levels observed in ddY-H mice were not caused by
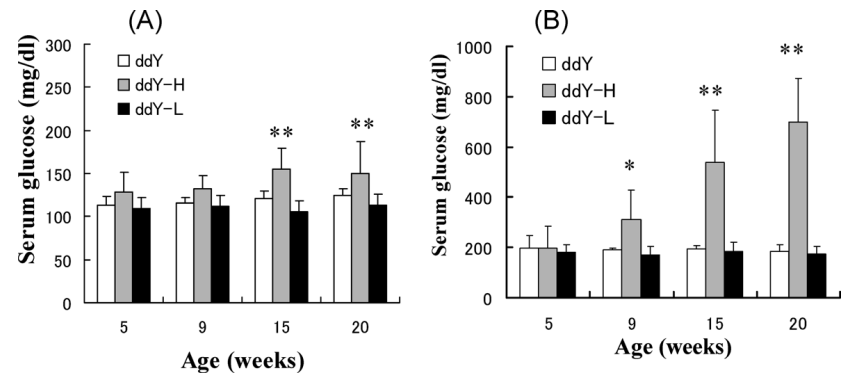

Fig. 2. Serum Glucose Level in Fasted Mice (A) and Refed Mice (B)

Mice fasted for $12 \mathrm{~h}$ (21:00 - 9:00) were allowed access to standard chow pellets for $1 \mathrm{~h}$ and serum glucose level was measured. Each column plus bar represents mean \pm S.D. of eight mice. $* * p<0.01, * p<0.05 v s$. ddY.
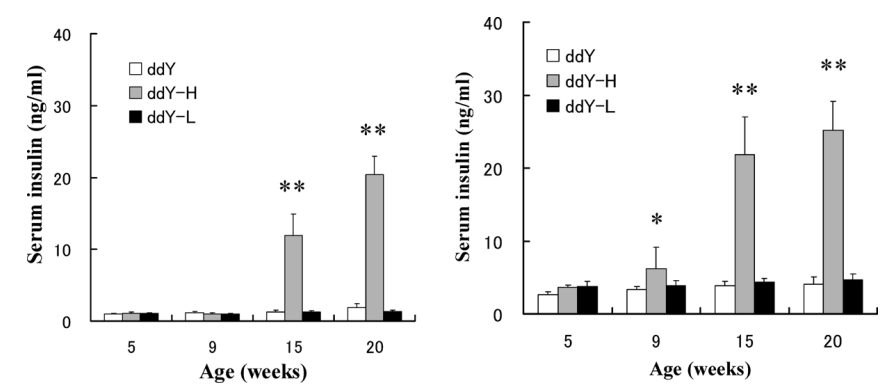

Fig. 3. Serum Insulin Level in Fasted Mice (A) and Refed Mice (B)

Mice were treated as described in Fig. 2, and serum insulin level was measured. Each column plus bar represents mean \pm S.D. of eight mice. $* * p<0.01, * p<0.05 v s$. ddY

differences in dietary intake. As for the ddY-H mouse, it was confirmed that normoglycemia was maintained in juvenile mice and serum glucose level was spontaneously elevated in mature mice.

Serum Insulin after Maintaining with Standard Chow Pellets Serum insulin levels at fasting and refeeding are shown in Fig. 3. There were no significant differences between ddY and ddY-L mice at all ages. However, serum insulin levels of ddY-H mice after fasting and refeeding were significantly elevated compared with other mice from 15 and 9 weeks of age, respectively. This suggests that the hyperglycemia induced in ddY-H mice after fasting and refeeding was not due to a defect in insulin secretion, but rather a decrease in insulin sensitivity.

Glucose Tolerance Test The glucose-loading test was performed to ensure that insulin resistance was induced in maturing ddY-H mice. Alterations in serum glucose levels and the area under the concentration-time lines $(A U C)$ are shown in Figs. 4 and 5. Alterations in serum glucose levels after glucose-loading in ddY and ddY-L mice were not influenced by maturing. However, serum glucose levels after glucose-loading in ddY-H mice at 12 and 15 weeks of age were higher than those in younger ddY-H mice, and ddY and ddY$\mathrm{L}$ mice of the same age, indicating that glucose tolerance was impaired in ddY-H mice at 12 and 15 weeks of age.

Insulin Sensitivity and Insulin Resistance Insulin sensitivity and insulin resistance were calculated in fasted mice at 15 weeks of age (Fig. 6). Insulin sensitivity was decreased and insulin resistance was increased in ddY-H mice compared with ddY mice, indicating that insulin resistance was induced in ddY-H mice at 15 weeks of age. However, urinary sugar level was not observed in all mice. On the other hand, 

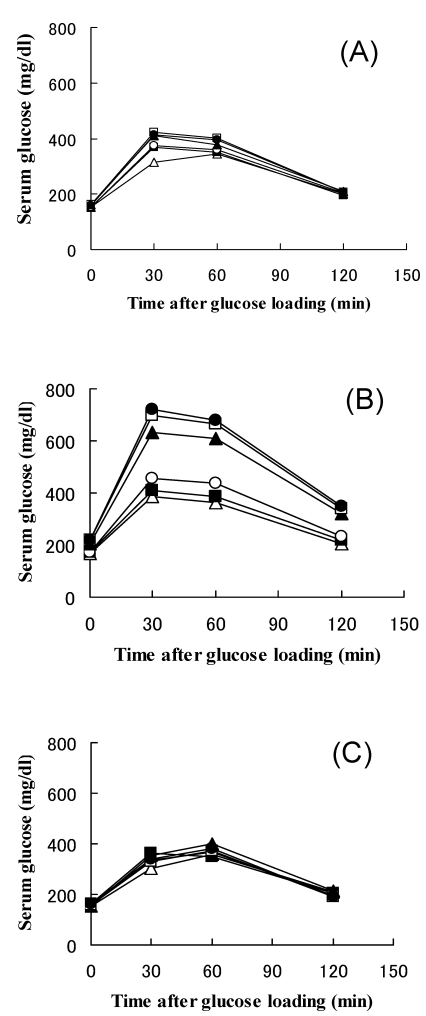

Fig. 4. Alterations in Serum Glucose Level after Glucose Loading in ddY (A), ddY-H (B) and ddY-L (C) Male Mice

Five- $(\triangle)$, seven- $(\mathbb{\square})$, nine- $(\bigcirc), 12-(\Delta), 15-(\square)$, and 20- $(\bullet)$ week-old mice fasted for $12 \mathrm{~h}(21: 00-9: 00)$ were orally administered glucose $(3 \mathrm{~g} / \mathrm{kg})$. Serum glucose level was measured after $0,30,60$ and $120 \mathrm{~min}$.

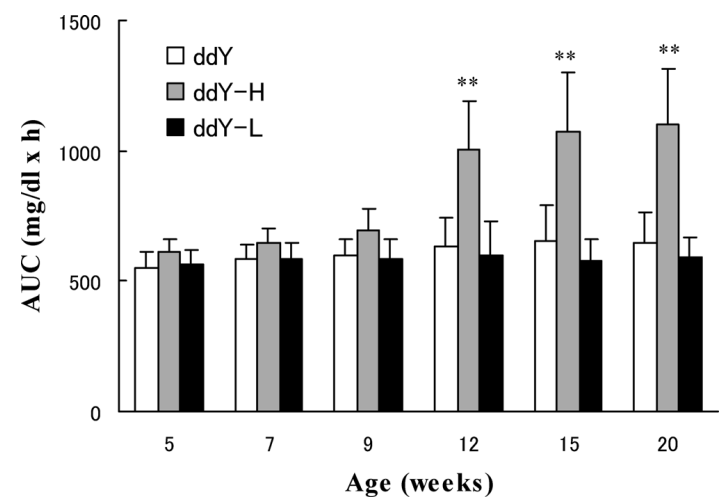

Fig. 5. $A U C$ in Glucose Tolerance Test

$A U C$ was calculated from data in Fig. 4. Each column plus bar represents mean \pm S.D. of eight mice. $* * p<0.01 v s$. ddY.
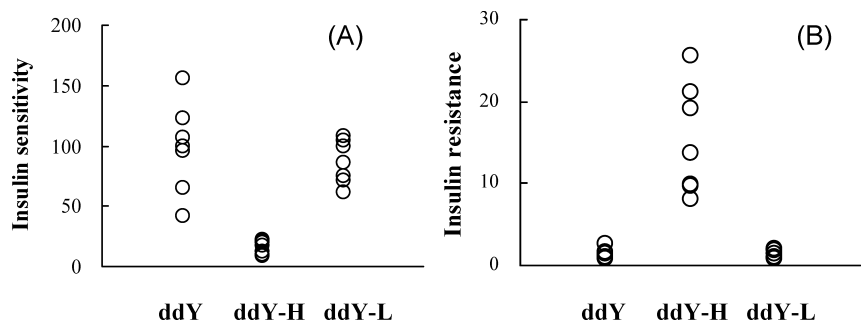

Fig. 6. Insulin Indexes in Male Mice at 15 Weeks of Age

Serum glucose and insulin levels were measured in mice fasted for $12 \mathrm{~h}(21: 00$ 9:00). Insulin sensitivity (glucose $(\mathrm{mg} / \mathrm{dl}) /$ insulin $(\mathrm{ng} / \mathrm{dl})(\mathrm{A})$ and insulin resistance (glucose $(\mathrm{mg} / \mathrm{dl}) \times$ insulin $(\mathrm{ng} / \mathrm{dl}) / 100)(B)$ were calculated. insulin ensitivity and insulin resistance were similar between ddY-L and ddY mice. The variation in insulin sensitivity within ddY-L mice was much smaller than that in ddY mice, suggesting that ddY-L mice have relatively equalized sensitivity.

Observation of Diabetic Symptoms To verify the induction of diabetic symptoms with aging, mice were maintained with standard chow pellets ad libitum up to 27 weeks of age. Body weight was extremely high in ddY-H mice compared with ddY mice and was significantly lower in ddY-L mice (Table 1). Serum glucose levels in ddY-H mice were markedly high $(362 \pm 101 \mathrm{mg} / \mathrm{dl})$ compared with those in $\mathrm{ddY}$ mice $(169 \pm 31 \mathrm{mg} / \mathrm{dl})$ and ddY-L mice $(147 \pm 19 \mathrm{mg} / \mathrm{dl})$. Serum insulin levels were also higher in ddY-H mice $(27.3 \pm 5.38 \mathrm{ng} / \mathrm{ml})$ than in $\mathrm{ddY}$ mice $(2.5 \pm 1.03 \mathrm{ng} / \mathrm{ml})$ and ddY-L mice $(1.8 \pm 0.78 \mathrm{ng} / \mathrm{ml})$. The food intakes were $4.9 \pm$ $0.9 \mathrm{~g} / \mathrm{d}$ in ddY mice, $5.2 \pm 0.8 \mathrm{~g} / \mathrm{d}$ in ddY-H mice, and $4.8 \pm$ $0.9 \mathrm{~g} / \mathrm{d}$ in ddY-L mice, with no significant differences. Urine collected for $24 \mathrm{~h}$ was used to measure glucose, and levels higher than $1000 \mathrm{mg} / \mathrm{ml}$ were detected in all ddY-H mice, but not in ddY and ddY-L mice (Table 1). In ddY-H mice, water intake and urinary volume were increased and urinary $\mathrm{pH}$ decreased. These results suggest that diabetic symptoms are spontaneously induced in ddY-H mice with aging, without the need for special loading such as a high-fat diet.

Cross-Mating of ddY-H and ddY-L Mice To certify the possibility that the diabetic symptoms in ddY-H mice are genetically induced, glucose tolerance test was carried out in mice born by cross-mating ddY-H and ddY-L mice. As shown in Figs. 7 and 8, an impaired glucose tolerance was only observed in mice born from male ddY-H and female ddY-H mice, and not in the offspring of male ddY-H and female ddY-L mice or male ddY-L and female ddY-H mice. This result suggests that the diabetic symptoms in ddY-H mice are genetically induced and that the characteristics are reces-

Table 1. Urinary Excretion in Male Mice at 27 Weeks of Age

\begin{tabular}{llllr}
\hline \hline Strain & \multicolumn{1}{c}{$\begin{array}{c}\text { Body } \\
\text { weight }(\mathrm{g})\end{array}$} & $\begin{array}{c}\text { Urine volume } \\
(\mathrm{ml} / \mathrm{d})\end{array}$ & \multicolumn{1}{c}{$\mathrm{pH}$} & \multicolumn{1}{c}{$\begin{array}{c}\text { Urinary } \\
\text { sugar }\end{array}$} \\
\hline ddY & $54.76 \pm 2.25$ & $2.14 \pm 0.39$ & $7.0 \pm 0.32$ & $0 / 15$ \\
ddY-H & $61.48 \pm 2.25^{* *, \# \#}$ & $3.64 \pm 1.23^{* *}, \#$ & $6.3 \pm 0.41^{\# \#}$ & $\begin{array}{r}15 / 15 \\
\text { ddY-L }\end{array}$ \\
\hline $44.77 \pm 2.01^{* *}$ & $1.64 \pm 0.57^{*}$ & $7.8 \pm 0.42$ & $0 / 15$ \\
\hline
\end{tabular}

Mice were maintained in metabolic cages to obtain urine for $24 \mathrm{~h}$. Each value represents the mean \pm S.D. of 15 mice. $* * p<0.01$ and $* p<0.05 v s$. ddY, \# $p<0.01 v s$. ddYL.
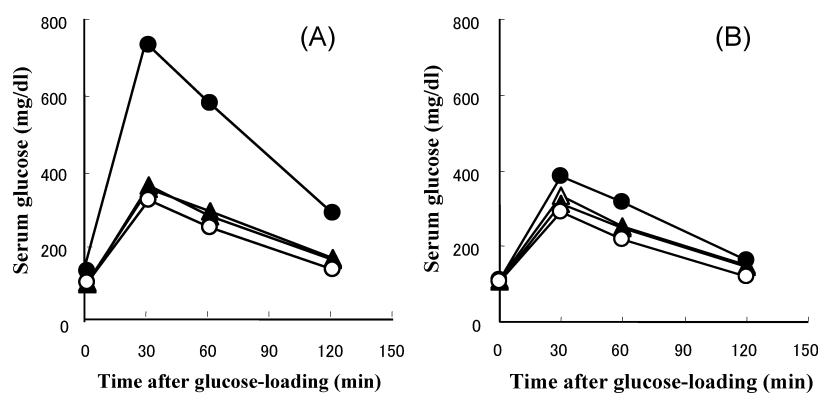

Fig. 7. Alterations in Serum Glucose Level after Glucose-Loading in ddY$\mathrm{H}(\bullet)$, ddY-LH $(\Delta)$, ddY-HL (ム) and ddY-L (O) Mice at 12 Weeks of Age

After fasting for $12 \mathrm{~h}(21: 00-9: 00)$, glucose tolerance test was carried out in fifteen male mice (A) and fifteen female mice (B). 


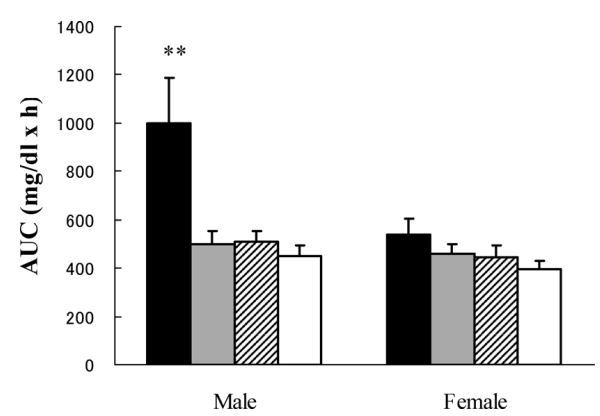

Fig. 8. AUC of Time-Serum Glucose Concentration Curve in Glucose Tolerance Test

$A U C$ was calculated from data of ddY-H (solid columns), ddY-LH (dotted columns), ddY-HL (shaded columns) and ddY-L (open columns) mice in Fig. 7. Each column plus bar represents mean \pm S.D. of 15 mice. $* * p<0.01 v s$. ddY-L.

sively inherited.

\section{DISCUSSION}

We have shown herein that sustained hyperglycemia is induced in ddY mice by refeeding with standard chow pellets after fasting for $48 \mathrm{~h} .{ }^{3)}$ However the serum glucose levels in refed mice vary widely, with the glucose level in ddY-H mice being markedly higher and that in ddY-L mice and relatively lower than that in the original ddY mice after fasting-refeeding. The striking differences in serum glucose levels between ddY-H and ddY-L mice were observed in male but not in female mice, and the characteristics are recessively inherited.

ddY-H mice maintained with standard chow pellets showed impaired glucose tolerance, and increased levels of serum glucose and insulin from 12-15 weeks of age under ordinary conditions without stress loading. At this time, diabetic symptoms such as the presence of urinary sugar were not found, but were detected in a few mice at 20 weeks of age (data not shown) and were observed in all 15 mice at 27 weeks of age. These results suggest that the impaired glucose tolerance of ddY-H mice is induced by maturing, followed by the appearance of diabetic symptoms with aging.

It is known that type 2 diabetes is induced by a defect in insulin secretion and a decrease in insulin sensitivity. ${ }^{2)}$ In some diabetic animal models, histological changes of pancreatic islets have been reported, including lymphocytic infiltration or fibrous formation in Otsuka Long-Evans Tokushima Fatty (OLETF) rats, ${ }^{8}$ vascularization in New Zealand obese (NZO) mice, ${ }^{12)}$ and degradation or necrosis in $\mathrm{Lep}^{\mathrm{ob}} / \mathrm{Lep}^{\mathrm{ob}}$ mice. ${ }^{13,14)}$ In ddY-H mice, severe changes in pancreatic islets such as inflammation and fibrous formation were not found, although hypertrophy and vacuolation were observed in a few mice at 15 weeks of age (data not shown). Since increased serum insulin levels were observed at 27 weeks of age, a defect in insulin secretion is not likely to contribute to diabetic symptoms in ddY-H mice.

Increases in serum insulin levels were observed from 15 weeks of age in fasted ddY-H mice and from 9 weeks of age in refed ddY-H mice, perhaps suggesting latent insulin resistance at 9 weeks of age. Furthermore, increases in serum insulin levels were timely in accord with increases in serum glucose levels and impaired glucose tolerance. Indices for insulin resistance of ddY-H mice at 15 weeks of age were markedly high compared with those of ddY and ddY-L mice.
Elevated serum insulin level is believed to be induced by the compensation of decreased insulin sensitivity, as observed in other diabetic mice models and type 2 diabetic patients. Repeated increases in serum insulin level and resultant decreases in target organ insulin sensitivity result in the slow enhancement of insulin resistance and the onset of diabetic symptoms. Our findings suggest that decreased insulin sensitivity in target organs might be attributed to impaired glucose tolerance and diabetic symptoms in ddY-H mice.

Suzuki et al. detected urinary sugar in six obese mice at $25-28$ weeks of age in their ddY-SPF colony. ${ }^{15)}$ Through repeated inbreeding using the indices of body mass and urinary sugar, they developed both Tsumura, Suzuki, Obese Diabetic mice (TSOD, obese and with urinary sugar) and Tsumura, Suzuki, Non Obesity mice (TSNO, without). TSOD mice have a tendency for obesity, hyperglycemia, and hyperinsulinemia, and develop diabetic symptoms by maintaining with the same chow pellets as in our experiments. Since these symptoms were only observed in male mice, there are many similarities between the characteristics of TSOD and ddY-H mice. A genome-wide screening identified three quantitative trait loci linked to glucose homeostasis and body weight in TSOD. ${ }^{16)}$ The major genetic determinant of blood glucose levels was identified on chromosome 11; two independent quantitative trait loci involved in controlling body weight were found on chromosomes 1 and 2, and one involved in controlling blood insulin level was identified on chromosome 2. They concluded that the diabetes mellitus-like symptoms of TSOD mice might be induced by a combination of these genetic loci.

ddY-H and TSOD mice are separated independently by different indices. However, both mice were derived from ddY mice, and developed common symptoms such as increases in body weight and levels of serum glucose, serum insulin and urinary sugar by maintaining with standard chow pellets. Although the genetic background of ddY-H mice is as yet unclear, some differences in characteristics between the mice are confirmed. TSOD mice develop severe glycosuria and increase their food and water intakes at the early pre-breeding age of 8 weeks. ${ }^{15)}$ ddY-H mice, by contrast, showed induced insulin resistance at $12-15$ weeks of age and glycosurea around 20 weeks of age. Therefore, the propagating ability was not changed from the original ddY mice (data not shown), demonstrating high utility as an experimental model animal. In TSOD mice, serum triglycerides and cholesterol levels were significantly high compared with controls. ddY-H mice showed significantly high serum triglycerides levels but low serum cholesterol levels compared with ddY and ddY-L mice (data not shown). These differences suggest that ddY-H and TSOD mice are different strains.

On the other hand, we have no evidence for differences between ddY-L and TSNO mice, with normal serum glucose and insulin levels and normal glucose tolerance being almost the same. However, individual variations, especially with regard to insulin indices and serum glucose level after refeeding, were smaller in ddY-L mice than in ddY mice. Urinary sugar was not detected in ddY-L mice with aging. ddY-L mice were derived from the same colony as ddY-H mice. These results demonstrate that ddY-L mice are normal mice without diabetic symptoms in contrast to ddY-H mice.

Type II diabetes in humans is a multifactorial disorder de- 
termined by environmental factors and genetic background, and is induced after middle age. ddY-H mice developed insulin resistance at $12-15$ weeks of age and showed diabetic symptoms after six months. It may also be said that the ddY$\mathrm{H}$ clinical condition resembles the onset of human clinical diabetes mellitus, rather than a disease of sudden progression. Furthermore, ddY-H mice show good propagating ability and are useful for long-term experiments such as the study of the mechanism of diabetic onset and the development of preventive medicine. In conclusion, ddY-H mice are a useful diabetic animal model with a spontaneous induction of diabetic symptoms without the need for loading such as nutritional stress; ddY-L mice are a good contrast control animal.

Acknowledgments This work was supported in part by a Grant-in-Aid for General Scientific Research (No. 16659040) from the Ministry of Education, Culture, Sports, Science and Technology of Japan. The authors are grateful to Dr. Kazuhiko Matsumoto, Torii Pharmaceutical Co., Ltd. for advising the statistical analysis.

\section{REFERENCES}

1) Taylor S. I., Cell, 97, 9-12 (1999).

2) Nakagawa C., Mineo I., Tarui S., Nippon Rinshou, 54, 2649-2656 (1996).

3) Herberg L., Coleman D. L., Metabolism, 26, 59-99 (1977).

4) Hummel K. P., Dickie M. M., Coleman D. L., Science, 153, 11271128 (1966).

5) Nakamura M., Yamada K., Diabetologia, 3, 212-221 (1967).

6) Goto Y., Kakizaki M., Masaki N., Tohoku J. Exp. Med., 119, 85-90 (1976).

7) Zuker L. M., Ann. N.Y. Acad. Sci., 131, 447-458 (1965).

8) Kawano K., Hirashima T., Mori S., Kurosumi M., Natori T., Diabetes, 41, 1422-1428 (1992).

9) Maeda T., Sakita R., Kaihatsu T., Miwa M., Biol. Pharm. Bull., 24, $950-953$ (2001).

10) Matthews D. R., Hosker J. P., Rudenski A. S., Naylor B. A., Treacher D. F., Turner R. C., Diabetologia, 28, 412-429 (1985).

11) Caro J. F., J. Clin. Endocrinol. Metab., 73, 691-695 (1991).

12) Bielschowsky M., Bielschowsky F., Aust. J. Exp. Biol. Med. Sci., 34, 181-198 (1956).

13) Like A. A., Chick W. L., Diabetologia, 6, 207-215 (1970a).

14) Like A. A., Chick W. L., Diabetologia, 6, 216-242 (1970b).

15) Suzuki W., Iizuka S., Tabuchi M., Funo S., Yanagisawa T., Kimura M., Sato T., Endo T., Kawamura H., Exp. Animal, 48, 181-189 (1999).

16) Hirayama I., Yi Z., Izumi S., Arai I., Suzuki W., Nagamachi Y., Kuwano H., Tekeuchi T., Izumi T., Diabetes, 48, 1183-1191 (1999). 